\title{
Am-241 incineration measurements with activation method in the QUINTA neutron field
}

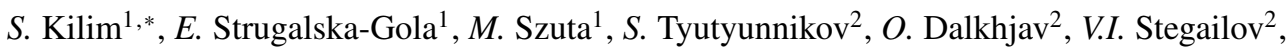 \\ I.A. Kryachko ${ }^{2}$, J.H. Khushvaktov ${ }^{2}$, A.G. Shakun ${ }^{2}$, F.B. Sagimbaeva ${ }^{2}$, and A.S. Balandin ${ }^{2}$ \\ ${ }^{1}$ National Centre for Nuclear Research, 05-400 Otwock-Świerk, Poland \\ ${ }^{2}$ Joint Institute for Nuclear Research, 141980 Dubna, Russia
}

\begin{abstract}
Am-241 sample was irradiated in spallation neutrons produced in ADS setup QUINTA at the JINR in Dubna. The energy was $660 \mathrm{MeV}$ in the proton beam. The incineration study method was based on gamma-ray spectrometry. During the analysis of the spectra, several fission products were identified. Fission product activities yielded the number of fissions. Nevertheless, the lines are assumed to belong to the neutron capture product covered by parasitic Np-238 decay lines. The Np-238 lines as a result of neutron capture by Np-237 made impossible to determine the number of captures in Am-241.
\end{abstract}

\section{Introduction}

Americium is one of 15 actinides produced artificially in power reactors as a by-product of energy production. It is $\alpha$-active with half-life time 432.2 year [1]. It is neutron capture cross section - Fig. 1 - dominates much the fission one in $0-1 \mathrm{MeV}$ energy range, i.e. in the most available energy in the present-day power reactors. As a consequence, the neutron capture induces the production of other actinides. The newly produced actinides affect the neutron balance so much that a fuel campaign is shortened. A neutron energy region exists, at approximately $1 \mathrm{MeV}$ and above, where fission of Am-241 dominates the radiative capture. To prevent/eliminate long-lasting activity/actinides, one needs a fast neutron source like a fast reactor or the accelerator-driven system (ADS).

The Am-241 incineration results presented here have been taken from the experiment carried out in November 08, 2014 and December 04, 2015 at the ADS-type setup QUINTA at JINR in Dubna, Russia. The QUINTA setup was irradiated with the $0.66 \mathrm{GeV}$ proton beam.

\section{Experiment and data description}

The core of the QUINTA setup - Fig. 2 - consisted of aluminum-cladded elements of natural uranium arranged in five hexagonal sections. The total weight of the uranium was 500 $\mathrm{kg}$. The uranium core was surrounded by a $10 \mathrm{~cm}$-thick lead shield. For more details of the QUINTA setup construction see [2]. The accelerated beam of $660 \mathrm{MeV}$ protons impinged on the uranium core causing nuclei spallation and the production of fast neutrons. The Am-241 sample was located in a side window about $20 \mathrm{~cm}$ from the axis of the beam. The spallation

\footnotetext{
*e-mail: stanislaw.kilim@ncbj.gov.pl
} 


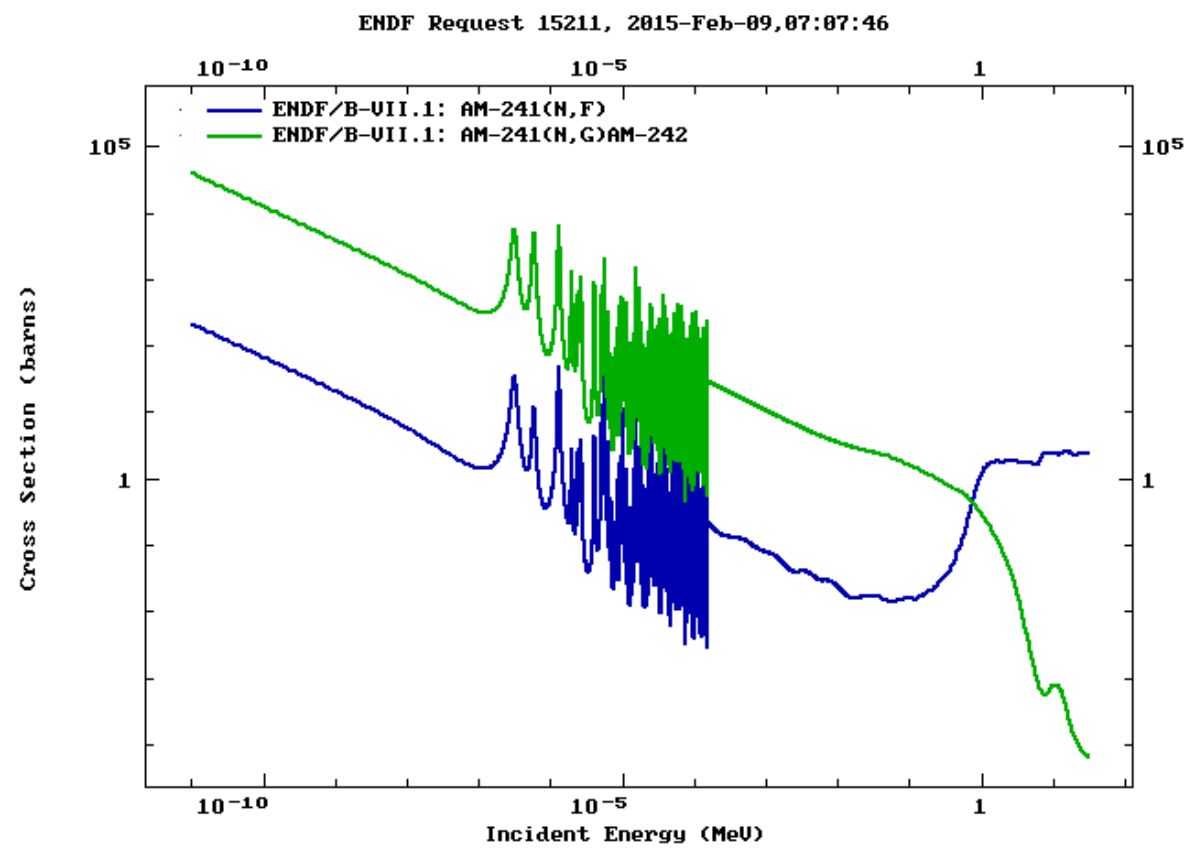

Figure 1. Am-241 neutron-caused fission (dark blue) and capture (green) cross section dependence on Energy

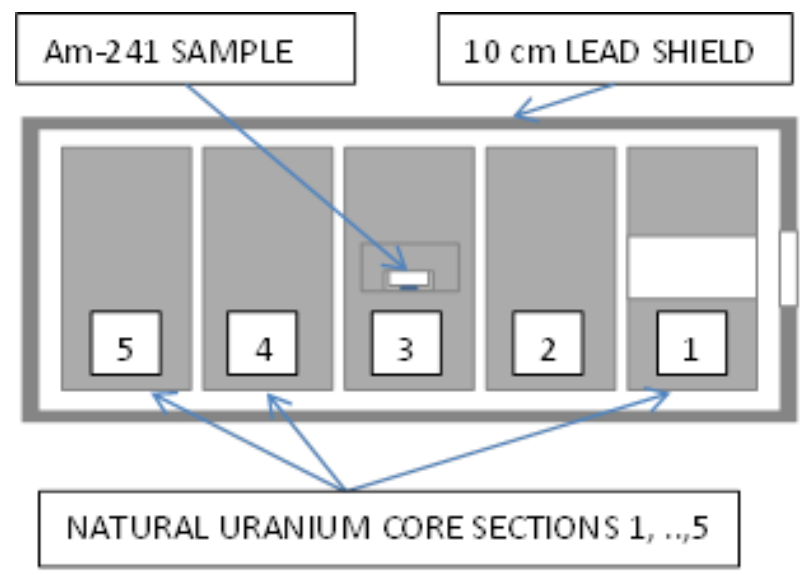

Figure 2. QUINTA setup vertical-axial cross section

neutrons caused U-238 fission which generated a number of neutrons while converting into the Am sample. The Am-241 sample was formed of a disk $21 \mathrm{~mm}$ in diameter encapsulated in a U-shaped casing. The gamma spectrum of the sample was measured several times by a CANBERRA GR1819 spectrometer. The spectroscopy filters were made of $\mathrm{Pb}, \mathrm{Cd}$ and $\mathrm{Cu}$ to reduce X-ray and low-energy gamma activity. Altogether it was composed of a 11.5 
mm-thick $\mathrm{Pb}$ filter, in addition to $1 \mathrm{~mm}$ of $\mathrm{Cd}$ and $1 \mathrm{~mm}$ of $\mathrm{Cu}$. Then, the following gamma spectrum analysis aimed to identify gamma peaks as well as calculate their areas and parent isotopes. Co-60, Ba-133, Cs-137, Eu-152 and Th-228 samples were used as calibration sources for gamma spectrometer efficiency, $\varepsilon_{p}$. Generally some peaks stem from Am-241 fission products and the others from the neutron capture product. The final measurements identified the number of Am-241 nucleus fissions and the nuclei which absorbed neutrons. They were simply calculated from the respective peak areas according to the formula:

$$
R_{f \gamma}=\frac{S_{\gamma}}{m \cdot \phi \cdot \gamma_{f} \cdot \varepsilon_{p} \cdot I_{\gamma}} \cdot \frac{\lambda \cdot t_{\text {irr }}}{\left(1-e^{-\lambda \cdot t_{\text {irr }}}\right)} \cdot \frac{e^{\lambda \cdot t_{+}}}{\left(1-e^{\left.-\lambda \cdot t_{\text {real }}\right)}\right.} \cdot \frac{t_{\text {real }}}{t_{\text {live }}},
$$

$R_{f \gamma}$ - actinide fission rate, per beam particle and per gram,

$\gamma$-gamma line index,

$f$ - reaction index ( $\mathrm{f}=$ fission $)$,

$S_{\gamma}$ - gamma peak area,

$\gamma_{f}$-isotope production yield, \% [5],

$m$ - activation sample mass, $\mathrm{g}$,

$\varepsilon_{p}$ - gamma spectrometer efficiency,

$I_{\gamma}$ - gamma line intensity, $\%$,

$\phi-$ (deuteron/proton/C6+) integral number,

$\lambda$-isotope decay constant,

$t_{+}-$cooling time,

$t_{\text {irr }}$ - irradiation time,

$t_{\text {real }}$ - real time of measurement,

$t_{\text {live }}$ - live time of measurement.

In the simplest case the isotopes are produced directly from fission (like Sr-92, I-133 and I-135) or capture, and the beam intensity is constant. There are some lines - like 658, 667, 772.6 and $954 \mathrm{keV}$ - which behave in a more complicated way described in [3].

Tab. 1 shows all identified gamma lines and their data (isotope source, half-life, intensity and respective FP yield).

Data averaging was performed over two steps - averaging over measurements for each isotope and then over isotopes or gamma lines in the capture case (Fig. 3).

Neutron capture product lines were calculated according to the formula similar to (1) but coefficient $\gamma_{f}$ was omitted.

\subsection{Results}

The results are presented in a selective way to illustrate some work out stages.

Fig. 3 presents the experiment of November 08, 2014, the Am-241 fission rate partial results, i.e. each series represents one fission product. Punctual marks represent values for one isotope (FP), for one measurement. The solid line represents the average value, averaged the all measurements and over all isotopes. Dashed lines represent the $\pm \sigma$ (standard deviation) range.

Fig. 4 presents the experiment of December 04, 2015, the Am-241 fission rate results averaged over the all measurements. Punctual marks represent average values for one isotope (FP). The solid line represents the average value, averaged over all isotopes. Dashed lines represent the $\pm \sigma$ (standard deviation) range. The average Am-241fission rate was found to be $1.34(37) \times 10^{-5}$ fissions/g/beam particle.

Fig. 5 was supposed to present the experiment of November 08, 2014, Am-241 capture rate. The results were worked out with (1) like formula where the most important correction 
Table 1. Sample Am-241-identified gamma lines and their data

\begin{tabular}{|c|c|c|c|c|c|}
\hline E-gamma & Isotope & Source & $\mathbf{T}_{1 / 2}$ & $\begin{array}{l}\text { Fission } \\
\text { yield, } \%[4]\end{array}$ & I-gamma, $\%$ [5] \\
\hline 529.87 & ${ }^{133} \mathrm{I}$ & FP & $20.87 \mathrm{~h}$ & 5.8 & 87 \\
\hline 658.08 & ${ }^{97} \mathrm{Nb}^{*}$ & FP & $16.744 \mathrm{~h}$ & 4.54 & 98.23 \\
\hline 667.71 & ${ }^{132} I^{* *}$ & FP & $3.26 \mathrm{~d}$ & 4.03 & 98.7 \\
\hline 743.36 & ${ }^{97} \mathrm{Zr}$ & FP & $16.744 \mathrm{~h}$ & 4.54 & 93.6 \\
\hline 772.60 & ${ }^{132} I^{* * *}$ & FP & $3.26 \mathrm{~d}$ & 4.03 & 75.6 \\
\hline 954.55 & ${ }^{132} \mathrm{I}^{* *}$ & FP & $3.26 \mathrm{~d}$ & 4.03 & 17.6 \\
\hline 1131.51 & ${ }^{135} \mathrm{I}$ & FP & $6.57 \mathrm{~h}$ & 5.09 & 22.6 \\
\hline 1260.41 & ${ }^{135} \mathrm{I}$ & FP & $6.57 \mathrm{~h}$ & 5.09 & 28.7 \\
\hline 1383.93 & ${ }^{92} \mathrm{Sr}$ & FP & $2.66 \mathrm{~h}$ & 2.09 & 90 \\
\hline 923.98 & ${ }^{238} \mathrm{~Np}$ & $\mathrm{CP}$ & $2.117 \mathrm{~d}$ & $\mathrm{~N} / \mathrm{A}$ & 2.869 \\
\hline 962.77 & ${ }^{238} \mathrm{~Np}$ & $\mathrm{CP}$ & $2.117 \mathrm{~d}$ & $\mathrm{~N} / \mathrm{A}$ & 0.702 \\
\hline 984.45 & ${ }^{238} \mathrm{~Np}$ & $\mathrm{CP}$ & $2.117 d$ & $\mathrm{~N} / \mathrm{A}$ & 27.8 \\
\hline 1025.87 & ${ }^{238} \mathrm{~Np}$ & $\mathrm{CP}$ & $2.117 \mathrm{~d}$ & $\mathrm{~N} / \mathrm{A}$ & 9.65 \\
\hline 1028.54 & ${ }^{238} \mathrm{~Np}$ & $\mathrm{CP}$ & $2.117 d$ & $\mathrm{~N} / \mathrm{A}$ & 20.38 \\
\hline
\end{tabular}

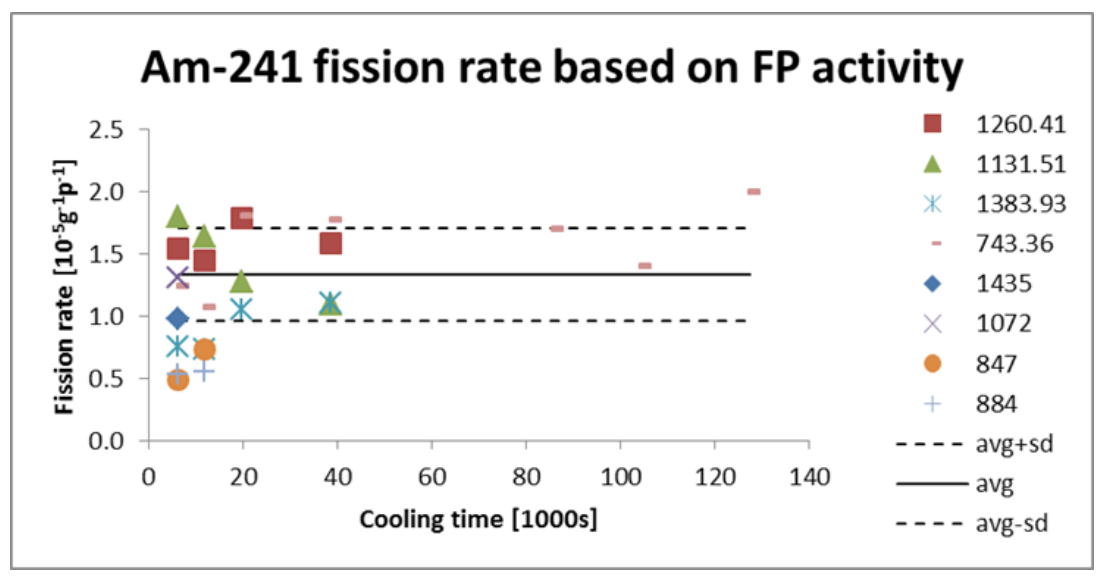

Figure 3. Am-241 fission rate measurement specific partial results

was for cooling time. In fact, it shows how complicated the problem is. At first, the lines supposedly belonging to the capture product isotopes are identical with the Np-238 ones i.e. with Np-237 neutron capture product ones. At second, the correction for cooling time yields a strange dependence on the cooling time. This dependence shows that there are two independent sources of 984 and $1028 \mathrm{keV}$ gamma lines. The first one with 2.117 day half life time and the next one with 141 year half life time. Am-241 related the decay/reaction scheme presented on the Fig. 6 explains what kind of processes cause the observed dependence.

The Am-241 sample used in the experiment was a rather old one: $10-15$ years old. As Am-241 undergoes $\alpha$-decay with 432.3 years of half life time it permanently produces Np237 - look at the upper channel of the reaction. During irradiation in the QUINTA the Np-237 captures the neutron yielding $\beta$-active $\mathrm{Np}$-238 isotope which has $2.117 \mathrm{~d}$ half-life time. 


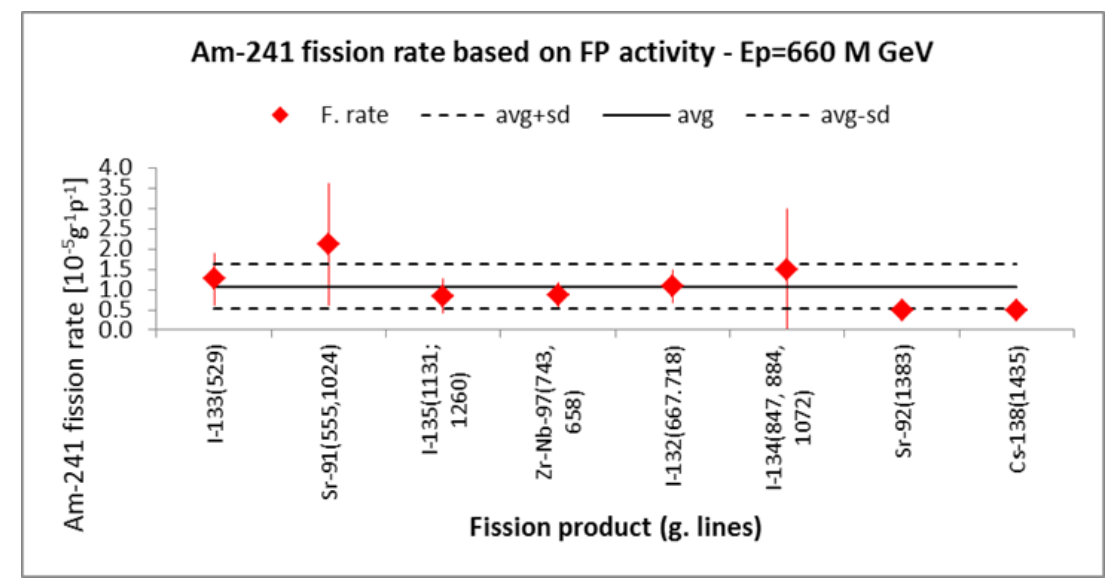

Figure 4. Am-241 fission rate FP isotope specific results

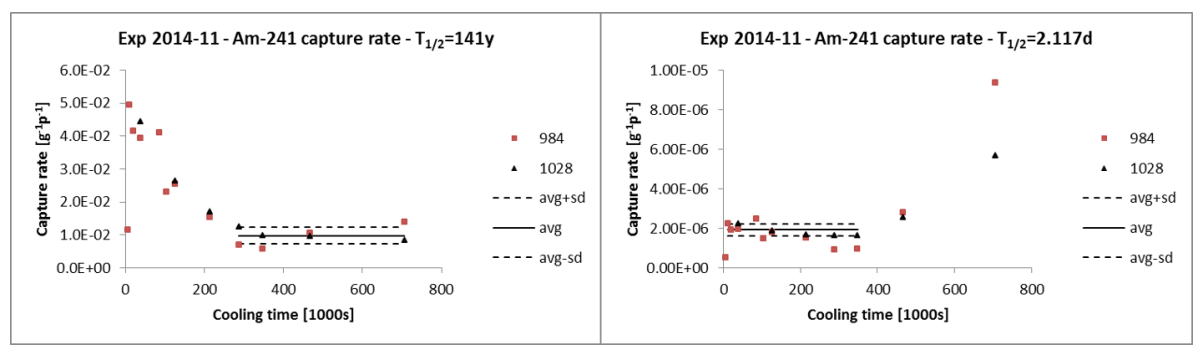

Figure 5. Line 984 and $1028 \mathrm{keV}$ corrected activity showing that there are two independent sources of the specified gammas

Another channel - Am-241(n, $\gamma)$ Am-242m( $\alpha$ )Np-238 - yields the same lines. Nevertheless, Am-242 with 141y half-life time dominates its observed decay time.

The Am-241 neutron capture channel - Am-241(n, $\gamma) A m-242(\beta$-)Cm-242 - yields the lines $561.1,605.13,897.33 \mathrm{keV}$ and more, this channel is specific, but their intensity is so low, that the lines have not been registered and the number of the captures has not been determinated. To overpass this problem one should use a very fresh Am-241 sample or apply a completely different method.

\section{Conclusions}

Two experiments have been carried out using a QUINTA-ADS-type setup to investigate the incineration potential of Am-241 in the left side window position. The ion beam consisted of protons $(0.66 \mathrm{GeV})$.

The activation and gamma spectrometry method was used.

Fission product of specific gamma lines and respective fission products were identified. Their activities yielded Am-241 fission rate $-1.34(37) \times 10^{-5}$ fissions/sample gram/beam particle. 


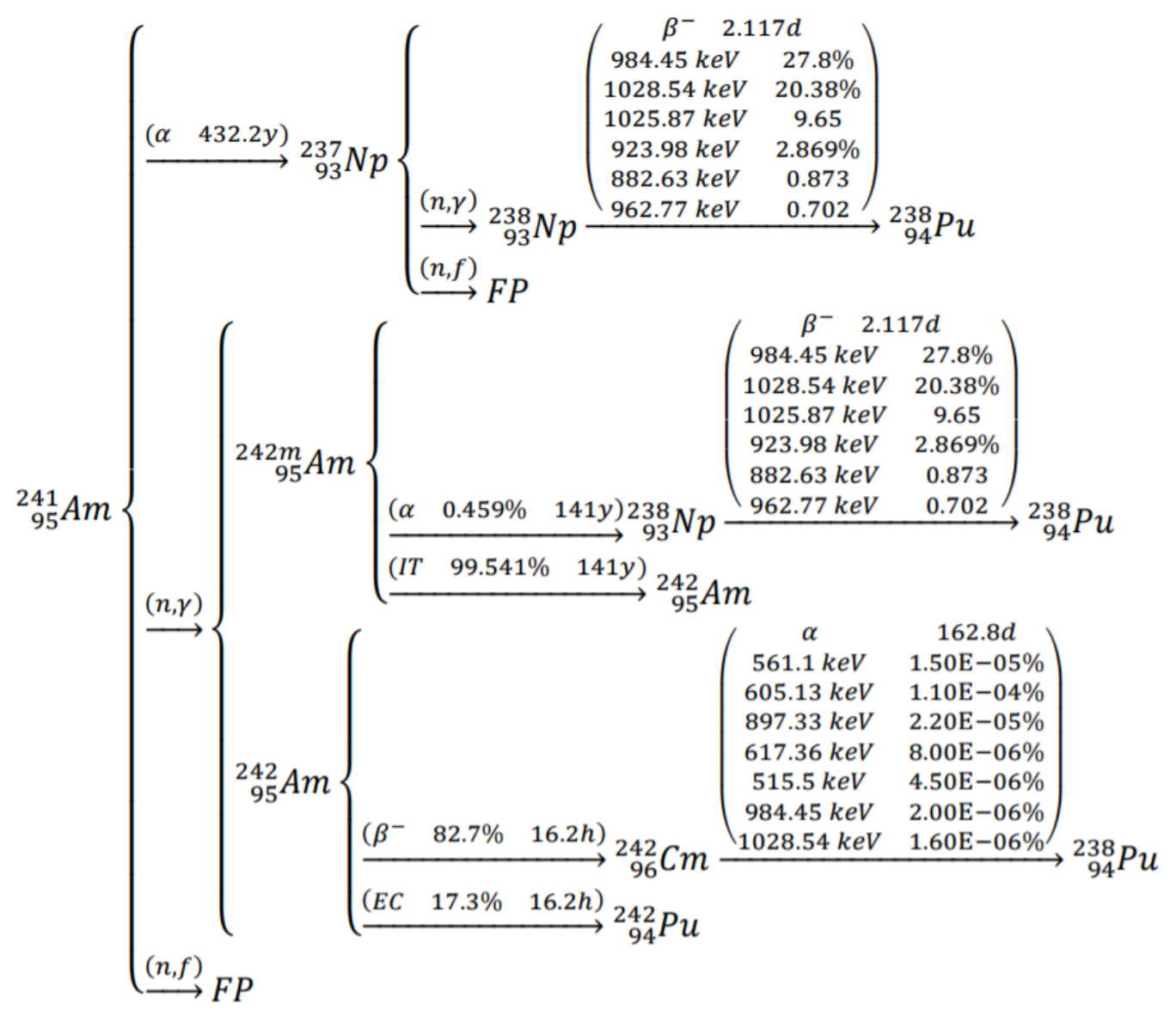

Figure 6. Am-241 related decay/reaction scheme

Am-241 capture of specific gamma lines were dominated by Np-238 $\beta$-decay lines making impossible to determinate the number of captures. The Am-241 activation method failed as the method to determinate the number of the captures.

Acknowledgments. The authors would like to thank the staff of the Nuclotron and Phasotron accelerators at the Joint Institute for Nuclear Research in Dubna, Russia, for providing access to the research facilities used in these experiments. 


\section{References}

[1] http://nucleardata.nuclear.lu.se/toi/listnuc.asp?sql=\&Z=93

[2] W. Furman et al., PoS Baldin ISHEPP XXI, 086 (2013)

[3] S. Kilim et al., Nukleonika 63(1), 17-22 (2018). doi:10.1515/nuka-2018-0003

[4] Evaluated Nuclear Data File (ENDF). https://www-nds.iaea.org/exfor/endf.htm

[5] R.B. Firestone, V.S. Shirley, C.M. Baglin, S.Y.F. Chu, J, Zipkin, The 8th edition of the Table of Isotopes, in Proceedings of the 9th International Symposium on Capture gammaray spectroscopy and related topics. V. 2 (Hungary, 1997) 